\section{P199 DOES AVERAGE VOLUME ASSURED PRESSURE SUPPORT (AVAPS) VENTILATION IMPROVE SAFETY IN MOTOR NEURONE DISEASE?}

TS Buttle, S Nathoo, J Kindred, S Banerjee. Medway Maritime Hospital, Gillingham, UK

\subsection{6/thoraxjnl-2015-207770.336}

Average volume-assured pressure support (AVAPS) is a novel way to deliver NIV. In this mode, a target tidal volume is set, and the device adjusts the pressure support to reach that volume. A particular potential benefit is that it may adapt to disease progression, as in patients with progressive Motor Neurone Disease. NICE guidance (2010) recommend follow up every 3 months. We propose to investigate if this new technology improves safety during the initial period of ventilator support.

Aim

1. To identify the trend in pressure support and hours of use of AVAPS ventilation in patients with ventilatory failure due to MND.

2. Look at compliance and tolerability on patients with AVAPS.

Methods Retrospective review of case notes and downloads from the ventilators of 6 patients identified to have started on AVAPS due to ventilatory failure secondary to MND. Average AHI, IPAP, EPAP, hours of use, compliance during first three months were reviewed.

Results There was no significant change in IPAP (Mean 14.78 at 1 month, 14.98 at 3 months) or EPAP (5.91 at 1 month, 6.57 at 3 months). Average use (6 hrs $44 \mathrm{~min}$ at one month rising to 8 hrs $48 \mathrm{~min}$ at three months) and compliance (percent greater than $4 \mathrm{~h} 77.6 \%$ at 1 month to $89.5 \%$ at 3 months) did show positive trends but did not reach significance.

\begin{tabular}{lll} 
Abstract P199 Table 1 & Summary of NIV usage & \\
\hline N & Month 1 & Month 3 \\
AHI & 6 & 6 \\
IPAP & 6.98 & 4.8 \\
EPAP & 14.78 & 14.98 \\
Avg use & 5.91 & 6.57 \\
Avg hrs when using & $06: 44$ & $08: 30$ \\
\% $>$ 4hrs & $06: 44$ & $08: 48$ \\
\hline
\end{tabular}

Conclusions This study shows an increase in average hours of use and compliance in the first 3 months of use. Tidal volumes and pressure support remain preserved. This initial data would suggest no benefit in providing the more expensive AVAPS machine compared to standard BiPAP S/T mode. Larger prospective studies looking at disease progression and ventilation usage in MND are warranted.

\section{P200 DOMICILIARY NOCTURNAL NIV IN COPD - STILL CONTROVERSIAL?}

J Barnacle, CME Longley, V Padmanaban, S Elkin, SAA Bloch. Imperial College Healthcare NHS Trust, London, UK

10.1136/thoraxjn-2015-207770.337
Introduction Despite strong evidence for the use of Non-invasive ventilation (NIV) in acute exacerbations of COPD resulting in decompensated type 2 respiratory failure (T2RF), the evidence for the long-term use of nocturnal NIV to prevent readmission or improve survival is controversial and has often been contradictory. Therefore clinicians are faced with the difficult question of what to do with COPD patients who are admitted with severe exacerbations requiring NIV and are considered at high risk of future decompensation. We hypothesised that domiciliary nocturnal NIV, established following an acute admission with decompensated T2RF delayed readmission.

Methods We performed a retrospective case note analysis of patients started on domiciliary NIV following acute admission to a busy central London acute trust. Indication for NIV and success of treatment were assessed. Time between admissions prior to establishing domiciliary NIV and time to $1^{\text {st }}$ readmission were compared.

Results 18 patients were identified from our database. (2 were excluded: 1 returned their machine immediately, the other never attended for any follow-up at our hospital.) To our knowledge the patients were not admitted to other hospitals in the year pre or post the index admission - the admission at which NIV was initiated. The mean age of the 16 remaining patients was $70 \pm$ 12 years; 9 were female, 8 male. Indication for NIV in 13 patients was COPD with resistant or recurrent T2RF, 1 had COPD plus sarcoidosis and the remaining 2 had COPD plus obesity hypoventilation. NIV was shown to be successful in reducing $\mathrm{pCO}_{2}$ between discharge and first follow up (mean reduction $0.84 \pm 1.17 \mathrm{kPa} \mathrm{p}=0.01$ ). There was a trend towards delayed $1^{\text {st }}$ readmission following initiation of NIV, when compared to the time between previous admission (Kaplan-meier survival analysis. $\mathrm{p}=0.09$ Figure 1).

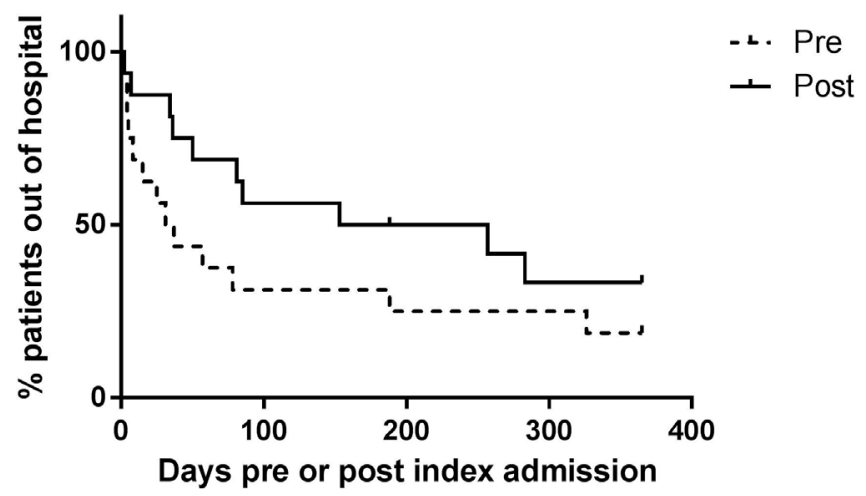

Abstract P200 Figure 1 Kaplan-meier curves of time pre or post index admission for patients $(n=16)$ started on domiciliary. Non-invasive ventilation (NIV) following acute admission with decompensated type 2 respiratory failure secondary to COPD. Difference between the curves suggests a trend towards delayed admission with initiation domiciliary NIV $p=0.09$

Conclusion Domiciliary NIV for high risk patients with decompensated T2RF in COPD is often used because of concerns of leaving the condition untreated when objectively NIV improved the patient's $\mathrm{pCO}_{2}$ furthermore there are no consistently ratified guidelines. The data presented here suggest that NIV may help to delay readmission to hospital. The results of ongoing randomised trials are eagerly awaited. 